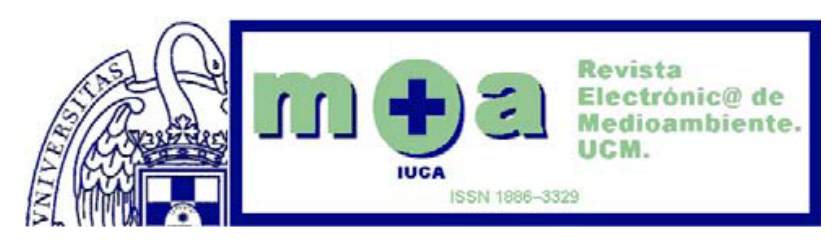

\title{
ESTUDIO ESTRUCTURAL DE UNA PANTALLA ACÚSTICA ECOSOSTENIBLE
}

\author{
Basilio MARTEL RODRÍgUeZ \\ basilio1martel@hotmail.com \\ María de la O Moreno Balboa \\ Universidad Alfonso X el Sabio \\ mmorebal@myuax.com \\ José Manuel Quintana Santana \\ Universidad de Las Palmas de Gran Canaria \\ josemanuel.quintana@ulpgc.es \\ Manuel Medina Molina \\ Universidad de Las Palmas de Gran Canaria \\ medina.0o@gmail.com
}

Recibido: 12 de noviembre del 2015

Enviado a evaluar: 13 de noviembre del 2015

Aceptado: 3 de diciembre del 2015

\section{RESUMEN}

El objeto de este estudio es comprobar la resistencia estructural de una pantalla acústica constituida con molido de poda de jardinería triturada, proveniente de los palmerales de Gran Canaria. Se ha utilizado arcilla como aglutinante, mediante su amasado con agua. Ambos materiales (molido y arcilla) se disponen en una proporción del $50 \%$. En la pantalla real construida se midió una resistencia mayor a $2.000 \mathrm{~kg} / \mathrm{m}^{2}$ y es estructuralmente estable. Mediante la simulación a partir del software SOLIDWORKS se lleva a cabo la comprobación de la estabilidad y comportamiento, de la misma, en condiciones de viento y lluvia. Tras el análisis de los resultados del ensayo, podemos afirmar, que la pantalla, presenta buena estabilidad incluso con lluvia abundante.

Palabras clave: Pantalla acústica, ensayo, simulación, SolidWorks, resistencia, molido de podas, arcilla y palmerales. 


\title{
STRUCTURAL STUDY OF AN ECO-SUSTAINABLE NOISE BARRIER
}

\begin{abstract}
The object of the present study is to check the structural strength of a noise barrier made of ground gardening pruning, coming from the palm groves in Gran Canaria. Clay has been used as agglutinative material, mixing it with water. Both materials (pruning and clay) are used in equal proportions (50\% and $50 \%)$. For the real barrier built we have measured a strength which is superior to $2.000 \mathrm{~kg} / \mathrm{m}^{2}$ and we have checked that it is structurally stable. By means of the SOLIDWORKS simulation we check its stability and its behaviour in windy and rainy conditions. Following the analysis of the test results we can state that the barrier has good stability even in heavy rain conditions.
\end{abstract}

Keywords: Noise barrier, test, simulation, SolidWorks, resistance, gardening pruning, clay and palm groves.

\section{ÉTUDE STRUCTURELLE DÚN ÉCRAN ACOUSTIQUE ECOSOSTENIBLE}

\section{RÉSUMÉ}

L'objet de cette étude est la vérification de la résistance structurelle d'un écran acoustique réalisé avec taille moulue de jardinage, provenant des palmeraies de la Grande Canarie. Nous avons utilisé de l'argile en tant que matériel agglutinant, en la mélangeant avec de l'eau. Les deux matériels (la taille et l'argile) sont utilisés dans des proportions égales ( $50 \%$ et $50 \%$ ). Pour l'écran réel bâti on a mesuré une résistance supérieure à $2.000 \mathrm{~kg} / \mathrm{m}^{2}$ et il est structurellement stable. Au travers de la simulation en SOLIDWORKS on vérifie sa stabilité et son comportement dans des conditions de vent et de pluie. Suite à l'analyse des résultats de l'essai nous pouvons affirmer que l'écran présente une bonne stabilité même dans des conditions de pluie abondante.

Mots-clés: Écran acoustique, essai, simulation, SolidWorks, résistance, moulue de jardinage, argile et palmeraies.

\section{MATERIALES EMPLEADOS}

\subsection{ARCILLAS}

La arcilla está formada por partículas de tamaño inferior a 0,002 $\mathrm{mm}$ y está constituida por cadenas tetraédricas y octaédricas, unidas por enlaces covalentes débiles. Las moléculas de agua pueden introducirse entre las cadenas, lo que conlleva un aumento del volumen. Presentan una gran capacidad de retención de agua (Ibáñez, J.J., 2006).

Cuando un suelo de arcilla se mezcla con agua, la arcilla fluye como un semilíquido. Cuando se seca, el suelo se comporta como un material sólido plástico. 


\subsection{MOLIDO DE JARDINERÍA}

El molido de jardinería proviene en mayor medida de los palmerales, ya que Gran Canaria posee 250 palmerales y unas 43.000 palmeras adultas naturales o silvestres distribuidas en todos los municipios, con excepción de San Mateo y Valleseco, donde no se han detectado núcleos naturales relevantes en sus términos municipales. Si a esta cantidad sumamos los ejemplares más jóvenes (brízales), cerca de 20.000, contabilizamos unos 63.000 ejemplares de palmeras en las isla de Gran Canaria.

De los 250 palmerales, un número muy relevante se distribuye en la vertiente sur de las isla, ente los barrancos de Tirajana y Mogán. En esta área que comprende los municipios sureños de Mogán, San Bartolomé y Santa Lucía de Tirajana, se extienden 102 poblaciones naturales que suponen el $41 \%$ de todas las palmeras de Gran Canaria.

Por municipios destacan Las Palmas de Gran Canaria $\left(10,2 \mathrm{~km}^{2}\right)$, San Bartolomé de Tirajana $\left(8,7 \mathrm{~km}^{2}\right)$ y Santa Lucía de Tirajana $\left(7,56 \mathrm{~km}^{2}\right)$ constituyendo entre los tres municipios el $45,7 \%$ de la extensión de palmeras en la isla.

El palmeral que mayor área ocupa es el situado en Ingenio, barranco de Los Majanos, municipio de Ingenio, que alcanza los $1,38 \mathrm{~km}^{2}$ (Sosa, P.A. et alii, 2007)

No hay datos oficiales sobre la cantidad de poda de palmeras ni del resto de plantas.

El molido de jardinería ayuda a compactar la mezcla, se pretende aportar una solución a la excesiva cantidad de residuos generados en la localidad. Además, se ha comprobado mediante ensayos complementarios que el comportamiento de este sustrato frente a la atenuación del ruido es satisfactorio.

Figura 1. Tierra y triturado de jardinería empleados en la pantalla.
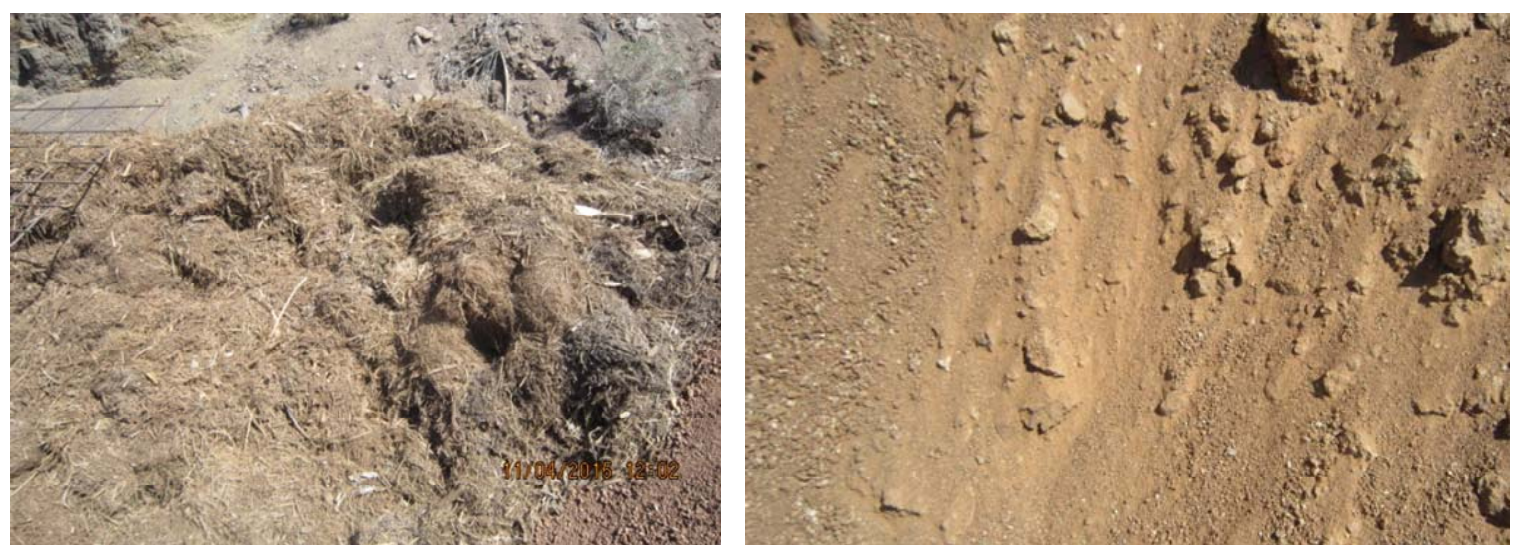

Fuente: Elaboración propia. 


\section{ESTADOS DE CONSISTENCIA}

El comportamiento de la pantalla, al igual que el de un suelo, está muy influenciado por su contenido en agua, sobre todo por el contenido arcilloso.

En función del grado de humedad, la pantalla puede adquirir diferentes estados de consistencia:

- Plástico: El material de la estructura es fácilmente moldeable, presentando grandes deformaciones con la aplicación de esfuerzos pequeños.

- Semisólido: La pantalla deja de ser moldeable, pues se quiebra y resquebraja antes de cambiar de forma. No obstante, no es un sólido puro, ya que disminuye de volumen si continúa perdiendo agua. Su comportamiento mecánico es aceptable.

- Sólido: En este estado el suelo alcanza la estabilidad, ya que su volumen no varía con los cambios de humedad. El comportamiento mecánico es óptimo [BAÑO00].

Figura 2. Origen de la cohesión en suelos arcillosos.
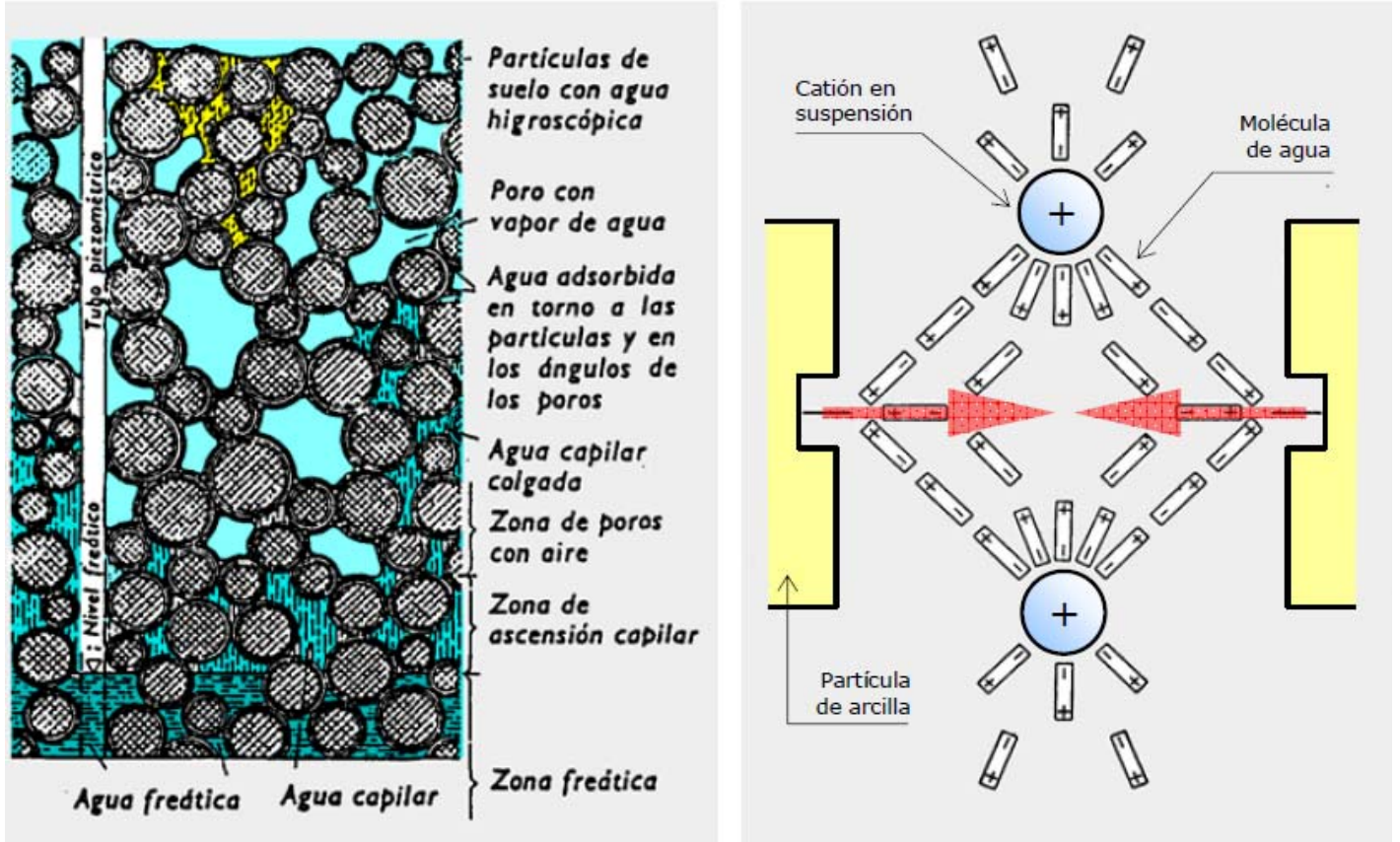

Fuente: Banon, L. et alii (2000).

\section{RESISTENCIA DEL SUELO}

Es importante conocer el comportamiento de la pantalla tanto a nivel teórico como real. La pantalla es una estructura resistente donde es importante conseguir estabilidad mecánica, distribución de las tensiones, que no se produzcan asientos excesivos incluso, el colapso.

Existen dos procedimientos de ensayo muy extendidos para determinar la resistencia mecánica del suelo: Los límites de Atterberg (NLT-105 y NLT106) y el equivalente de arena (NLT-113), si bien el primero es más preciso que el segundo (Banon, L. et alii, 2000). 


\section{LÍMITES DE ATTERBERG}

Atterberg fue pionero en determinar la relación existente entre el grado de plasticidad de un suelo con su contenido en agua o humedad, expresado en función del peso seco de la muestra. También fue él quien definió los cuatro estados de consistencia de los suelos y determinó los límites entre ellos, observando la variación de diferentes propiedades físicas y mecánicas.

De los límites anteriormente mencionados, interesa especialmente la determinación del límite plástico, ya que éstos presentan una alta deformabilidad del suelo y una drástica reducción de su capacidad portante. Afinando más todavía, el interés se centra en determinar el intervalo de humedad para el cual el suelo se comporta de manera plástica, es decir, su plasticidad (Banon, L. et alii, 2000).

\section{COMPACIDAD DEL SUELO}

La compacidad de un suelo es una propiedad importante, al estar directamente relacionada con la resistencia, deformabilidad y estabilidad; adquiere una importancia crucial en el caso de los terraplenes y todo tipo de relleno en general, en los que el suelo debe quedar lo más consolidado posible para evitar asientos.

\section{INFLUENCIA DE LA HUMEDAD}

En la compactación de suelos, la humedad juega un papel decisivo: mientras que un suelo seco necesita una determinada energía de compactación para vencer los rozamientos internos entre sus partículas, el mismo suelo ligeramente húmedo precisará un menor esfuerzo, ya que el agua se comporta como un agente lubricante formando una película alrededor de los granos y disminuyendo la fricción entre ellos.

Si aumentamos el porcentaje de agua, llegará un momento en el que ésta haya ocupado la totalidad de los huecos del mismo. Este hecho acarreará un aumento de volumen (dada la incompresibilidad del líquido elemento) y una mayor dificultad para evacuarlo del suelo, por lo que su compacidad disminuirá. De la anterior explicación, se deduce que existirá una humedad óptima con la que se obtenga una compacidad máxima, para una misma energía de compactación (Banon, L. et alii, 2000).

\section{RESISTENCIA DE LA PANTALLA}

Para comprobar la resistencia se midió en varios puntos: a alturas de 2 metros y 3 metros; transcurridas 24 horas, tras el llenado y compactado de la pantalla. Para cada punto de medida se homogeneizó una superficie circundante de $0,25 \mathrm{~m}^{2}$ mediante una mezcla a partes iguales de arcilla y de molido de jardinería, utilizando como aglutinante una proporción de $1 / 2$ de agua y aglutinado en hormigonera. Dicho elemento se sometió a una carga homogénea de $220 \mathrm{~kg}$. Como resultado se obtuvo una resistencia por $\mathrm{cm}^{2}$ de $\left(220 \mathrm{~kg} / 1089 \mathrm{~cm}^{2}\right) 0,20 \mathrm{~kg} / \mathrm{cm}^{2}$.

\section{JUSTIFICACIÓN DE DATOS}

A continuación se exponen los datos de partida, Figura 3. 
Figura 3. Propiedades mecánicas y físicas de rocas y suelos naturales.

\begin{tabular}{|c|c|c|c|c|}
\hline \multicolumn{5}{|c|}{ Rocas y suelos naturales } \\
\hline \multirow[b]{2}{*}{ Material } & \multicolumn{4}{|c|}{ HE } \\
\hline & $\rho\left[\mathrm{kg} / \mathrm{m}^{3}\right]$ & $\begin{array}{c}\lambda^{(1)} \\
{[W / m \cdot K]}\end{array}$ & $C_{p}[\mathrm{~J} / \mathrm{kg} \cdot \mathrm{K}]$ & $\boldsymbol{\mu}$ \\
\hline \multicolumn{5}{|l|}{ Rocas Ígneas } \\
\hline Basalto & $\begin{array}{c}2.700 \leq \rho \leq 3.0 \\
00\end{array}$ & 3,50 & 1.000 & 10.000 \\
\hline Granito & $\begin{array}{c}2.500 \leq \rho \leq 2.7 \\
00\end{array}$ & 2,80 & 1.000 & 10.000 \\
\hline Piedra pómez natural & $\rho \leq 400$ & 0,12 & 1.000 & 6 \\
\hline Roca natural porosa (por ej. Lava) & $\rho \leq 1.600$ & 0,55 & 1.000 & 15 \\
\hline Traquita, andesita & $\begin{array}{c}2.000 \leq \rho \leq 2.7 \\
00\end{array}$ & 1,10 & 1.000 & 15 \\
\hline Rocas o suelos sedimentarios & & & & \\
\hline Arcilla o limo & $\begin{array}{c}1.200 \leq \rho \leq 1.8 \\
00\end{array}$ & 1,50 & $1.670-2.500$ & 50 \\
\hline Arena y grava & $\begin{array}{c}1.700 \leq \rho \leq 2.2 \\
00\end{array}$ & 2,00 & $910-1.180$ & 50 \\
\hline Arenisca & $\begin{array}{c}2.200 \leq \rho \leq 2.6 \\
00\end{array}$ & 3,00 & & \\
\hline \multirow[t]{2}{*}{ Asperón } & $\begin{array}{c}1.900 \leq \rho \leq 2.5 \\
00\end{array}$ & 1,80 & 1.000 & 40 \\
\hline & $\begin{array}{c}1.300 \leq \rho \leq 1.9 \\
00\end{array}$ & 0,90 & 1.000 & 20 \\
\hline Caliza, muy dura & $\begin{array}{c}2.200 \leq \rho \leq 2.5 \\
90\end{array}$ & 2,30 & 1.000 & 200 \\
\hline Caliza, dura & $\begin{array}{c}2.000 \leq \rho \leq 2.1 \\
90\end{array}$ & 1,70 & 1.000 & 150 \\
\hline Caliza, dureza media & $\begin{array}{c}1.800 \leq \rho \leq 1.9 \\
90\end{array}$ & 1,40 & 1.000 & 40 \\
\hline Caliza, blanda & $\begin{array}{c}1.600 \leq \rho \leq 1.7 \\
90\end{array}$ & 1,10 & 1.000 & 25 \\
\hline Caliza, muy blanda & $\rho \leq 1.590$ & 0,85 & 1.000 & 20 \\
\hline Sílex & $\begin{array}{c}2.600 \leq \rho \leq 2.8 \\
00\end{array}$ & 2,60 & 1.000 & 10.000 \\
\hline \multicolumn{5}{|l|}{ Rocas metamórficas } \\
\hline Gneis, Pórfido & $\begin{array}{c}2.300 \leq \rho \leq 2.9 \\
00\end{array}$ & 3,50 & 1.000 & 10.000 \\
\hline Esquisto, Pizarra & $\begin{array}{c}2.000 \leq \rho \leq 2.8 \\
00\end{array}$ & 2,20 & 1.000 & 800 \\
\hline Mármol & $\begin{array}{c}2.600 \leq \rho \leq 2.8 \\
00\end{array}$ & 3,50 & 1.000 & 10.000 \\
\hline
\end{tabular}

Fuente: Instituto Eduardo Torroja de Ciencias de la Construcción, con la colaboración de CEPCO y AICIA (2010). 
Utilizando datos promedios entre tierra vegetal y arcilla y limo $(1.200 \leq$ densidad $\leq 1.925)$, promedio $1.562 \mathrm{~kg} / \mathrm{m}^{3}$ (Instituto Eduardo Torroja de Ciencias de la Construcción, 2010). Se toma un valor de 1.680 $\mathrm{kg} / \mathrm{m}^{3}$, superior al promedio, ya que el molido de jardinería es más consistente que el limo.

Para el caculo de la fuerza del viento se aplica la fórmula del CTE DB-SE-AE. Acciones en la edificación, articulo 3.3 (Ministerio de Fomento, 2009).

$q_{e}=q_{2} c_{e} c_{\mathrm{p}}$

donde $\mathrm{q}_{\mathrm{b}}$ es la presión dinámica del viento,

$c_{e}$ es el coeficiente de exposición, variable con la altura del punto considerado, en función del grado de aspereza del entorno donde se encuentra ubicada la construcción.

$c_{p}$ es el coeficiente eólico o de presión, dependiente de la forma y orientación de la superficie respecto al viento, y en su caso, de la situación del punto respecto a los bordes de esa superficie; un valor negativo indica succión.

De forma simplificada, como valor de la presión dinámica del viento en cualquier punto del territorio español, puede adoptarse $0,5 \mathrm{kN} / \mathrm{m}^{2}$.

En la Tabla 3.4 del capítulo 3.3.3 del CTE-DB-SE-AE se establecen los valores del coeficiente de exposición. Se ha elegido un valor de 2,2, ligeramente superior al correspondiente para terreno rural llano sin obstáculos ni arbolado de importancia a una altura de $3 \mathrm{~m}$, por ser una zona de viento racheado.

Para calcular el coeficiente eólico, la tabla 3.5 del capítulo 3.3.4 del CTEDB-SE-AE establece los valores para edificios de pisos en función de la esbeltez en el plano paralelo al viento. La esbeltez se ha calculado mediante la relación entre altura y profundidad máxima de la barrera, resultando un valor de 1,67. El coeficiente eólico resultante es igual a 0,8.

$$
q_{t}=0,5 \cdot 2,2 \cdot 0,8=880 \mathrm{~N} / \mathrm{m}^{2}
$$


Figura 4. Valores orientaticos de NSPT, resistencia a compresión simpley módulo de elasticidad de suelos. Datos para el cálculo de la elasticidad y el límite elástico.

\begin{tabular}{|c|c|c|c|}
\hline Tipo de suelo & $\mathbf{N}_{\text {SPT }}$ & $\mathbf{q}_{\mathbf{u}}\left[\mathbf{k N} / \mathbf{m}^{\mathbf{2}}\right]$ & $\mathbf{E}\left[\mathbf{M N} / \mathbf{m}^{\mathbf{2}}\right]$ \\
\hline $\begin{array}{c}\text { Suelos muy flojos o muy } \\
\text { blandos }\end{array}$ & $<10$ & $0-80$ & $<8$ \\
\hline Suelos flojos o blandos & $10-25$ & $80-150$ & $8-40$ \\
\hline Suelos medios & $25-50$ & $150-300$ & $40-100$ \\
\hline Suelos compactos o duros & $\begin{array}{c}50- \\
\text { Rechazo } \\
\text { Rechazo }\end{array}$ & $500-5.000$ & $500-8.000$ \\
\hline Rocas blandas & Rechazo & $\begin{array}{c}5.000- \\
40.000\end{array}$ & $\begin{array}{c}8.000- \\
15.000\end{array}$ \\
\hline Rocas duras & Rechazo & $>40.000$ & $>15.000$ \\
\hline
\end{tabular}

Fuente: Muelas Rodríguez, Á. (2015).

Se exponen a continuación los datos que verifican el cumplimiento del requisito que indica la Norma ISO 9613-2 7.4 (la densidad superficial debe ser mayor a $10 \mathrm{~kg} / \mathrm{m}^{2}$ ) (ISO 9613-2, 1996).

- Para suelos medios:

Límite de resistencia $150-300 \mathrm{kN} / \mathrm{m}^{2}$. Elasticidad $40-100 \mathrm{MN} / \mathrm{m}^{2}$.

- Para suelos blandos:

Límite de resistencia $80-150 \mathrm{kN} / \mathrm{m}^{2}$. Elasticidad $8-40 \mathrm{MN} / \mathrm{m}^{2}$.

Se han tomado los siguientes valores:

1. Pieza 1P (cara trasera $150 \mathrm{kN} / \mathrm{m}^{2}$ y $40 \mathrm{MN} / \mathrm{m}^{2}$ ).

2. Pieza 2P (cara trasera $225 \mathrm{kN} / \mathrm{m} 2$ y $70 \mathrm{MN} / \mathrm{m} 2$ ).

3. Pieza 3P (cara trasera $300 \mathrm{kN} / \mathrm{m} 2$ y $100 \mathrm{MN} / \mathrm{m} 2$ ).

4. Pieza 4P (cara delantera $150 \mathrm{kN} / \mathrm{m} 2$ y $40 \mathrm{MN} / \mathrm{m} 2$ ).

5. Pieza $5 \mathrm{P}$ (cara delantera $225 \mathrm{kN} / \mathrm{m} 2$ y $70 \mathrm{MN} / \mathrm{m} 2$ ).

6. Pieza 6P (cara delantera $300 \mathrm{kN} / \mathrm{m} 2$ y $100 \mathrm{MN} / \mathrm{m} 2$ )

7. Pieza 7P (cara trasera $80 \mathrm{kN} / \mathrm{m} 2$ y $8 \mathrm{MN} / \mathrm{m} 2$ )

8. Pieza $8 \mathrm{P}$ (cara delantera $80 \mathrm{kN} / \mathrm{m} 2$ y $8 \mathrm{MN} / \mathrm{m} 2$ )

9. Pieza 9P (cara delantera $300 \mathrm{kN} / \mathrm{m} 2$ y $100 \mathrm{MN} / \mathrm{m} 2$ )

\section{Simulación.}

Se ha realizado una simulación de la capacidad estructural (factor de seguridad y deformación) de la barrera con el software SOLIDWORKS. 


\section{Descripción.}

La pantalla posee las siguientes dimensiones:

Longitud: $4 \mathrm{~m}$.

Altura: $3 \mathrm{~m}$.

Sección: trapezoidal rectangular de $1,80 \mathrm{~m}$ de base y 0,55 $\mathrm{m}$ en la base superior.

Material: mezcla a partes iguales de arcilla y de molido de jardinería, utilizando como aglutinante una proporción de $1 / 2$ de agua.

Figura 5. Pantalla real sobre la que se han realizado pruebas de resistencia y medidas acústicas.

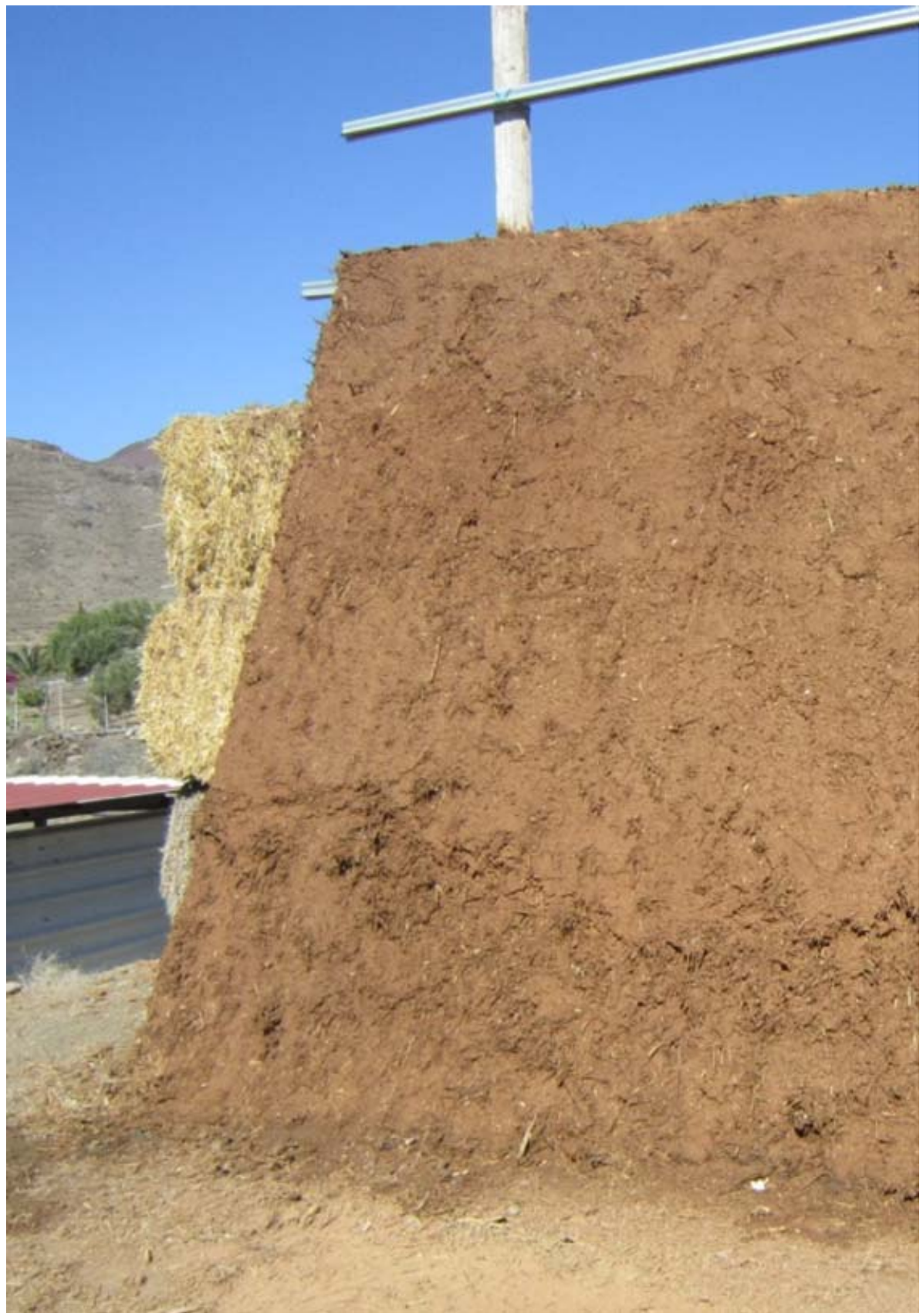

Fuente: Elaboración propia. 


\section{SIMULACIÓN DE LA PIEZA 1P.}

9.1. PROPIEDADES DEL MATERIAL

$$
\begin{array}{cc}
\text { Límite elástico: } & 150.000 \mathrm{~N} / \mathrm{m}^{2} \\
\text { Límite de compresión: } & 150.000 \mathrm{~N} / \mathrm{m}^{2} \\
\text { Módulo elástico: } & 4 \mathrm{e}+007 \mathrm{~N} / \mathrm{m}^{2} \\
\text { Coeficiente de Poisson: } & 0,35 \\
\text { Densidad: } & 1.680 \mathrm{~kg} / \mathrm{m}^{3}
\end{array}
$$

\subsection{RESULTADOS DEL ESTUDIO}

\subsubsection{DESPLAZAMIENTOS}

\section{Tipo \\ Mín. Máx. \\ Escala de deformación}

$0 \mathrm{~mm} \quad 2,30395 \mathrm{~mm}$

Nodo: 1 Nodo: 7.533

Desplazamiento resultante

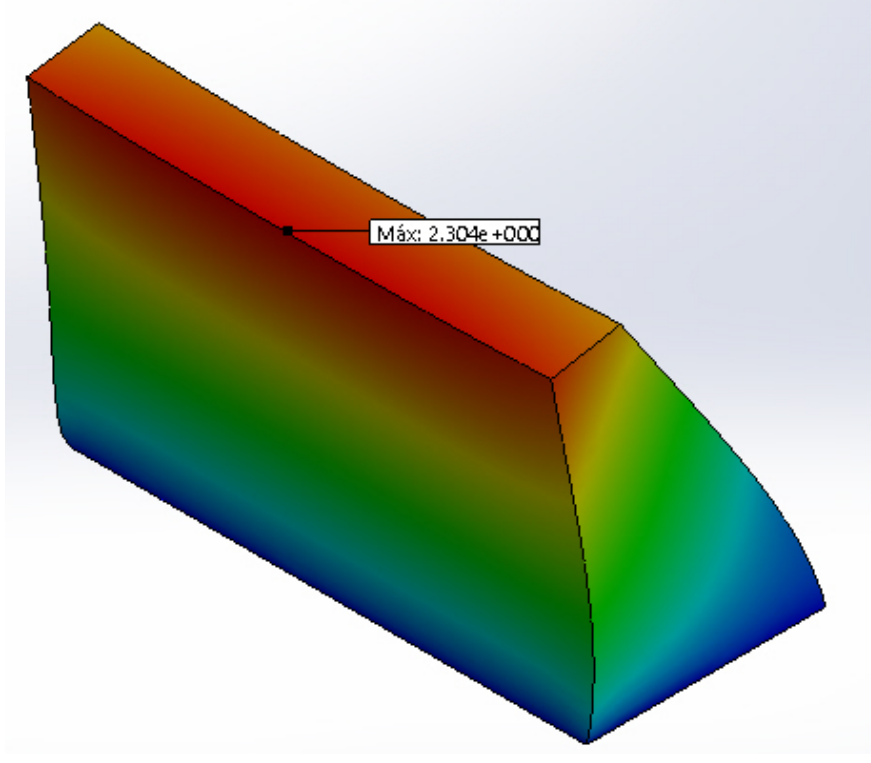
233,604 URES (mm)

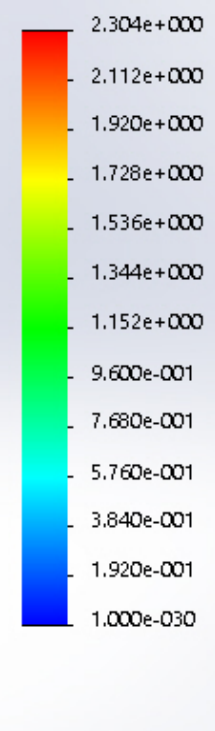

La deformación oscila entre 0 y 2,3 mm. 


\subsubsection{FACTOR DE SEGURIDAD}

Tipo

Automático
Mín. 1,94588 (1) 281,45

\section{Distribución de factor de seguridad}

FDS mín $=1,9$

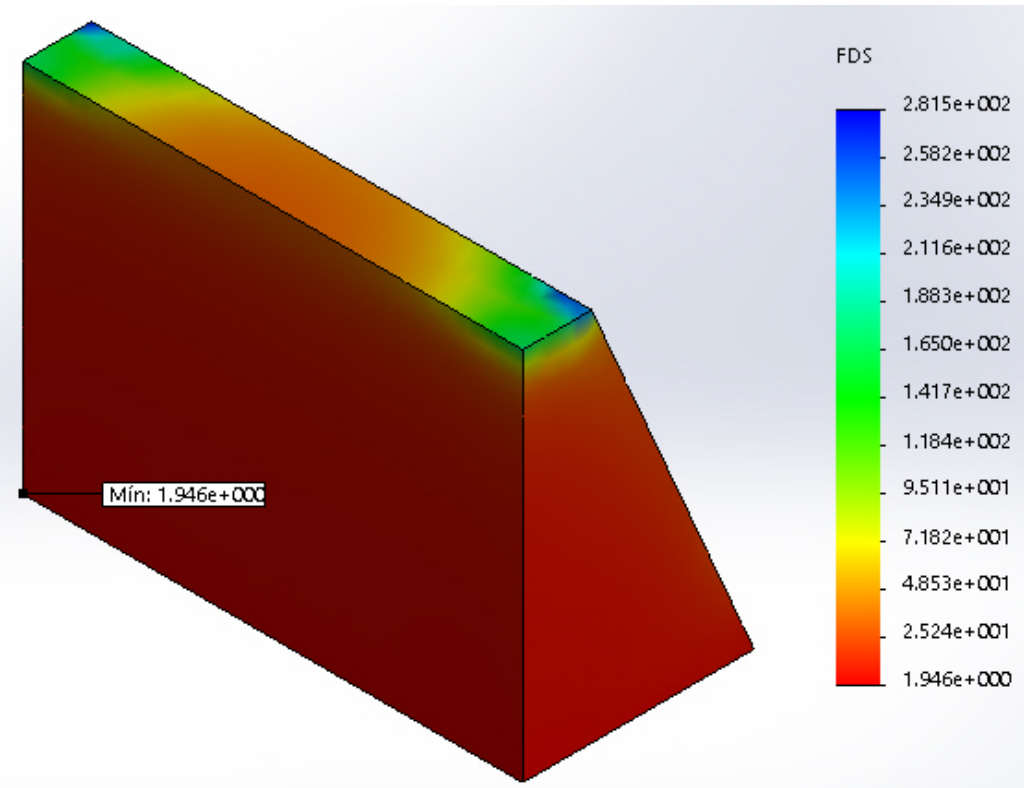

Las fuerzas aplicadas son 1,95 veces menores que el límite elástico.

\subsection{CONCLUSIÓN}

En base a los datos mostrados, podemos afirmar que la pantalla, en el caso más desfavorable, es capaz de resistir una fuerza de hasta $1.712,37 \mathrm{~N} / \mathrm{m}^{2}$ producida por viento siendo la fuerza estudiada, en base a la normativa aplicada, de $880 \mathrm{~N} / \mathrm{m}^{2}$, por lo tanto la resistencia es muy superior a la necesaria.

La deformación máxima a la fuerza aplicada es de 2,3 mm. 


\section{SIMULACIÓN DE LA PIEZA 2P.}

10.1. PROPIEDADES DEL MATERIAL

$$
\begin{array}{cc}
\text { Límite elástico: } & 230.000 \mathrm{~N} / \mathrm{m}^{2} \\
\hline \text { Límite de compresión: } & 225.000 \mathrm{~N} / \mathrm{m}^{2} \\
\text { Módulo elástico: } & 7 \mathrm{e}+007 \mathrm{~N} / \mathrm{m}^{2} \\
\text { Coeficiente de Poisson: } & 0,35 \\
\text { Densidad: } & 1.680 \mathrm{~kg} / \mathrm{m}^{3}
\end{array}
$$

\begin{tabular}{|c|c|c|c|}
\hline Tipo & Mín. & Máx. & Escala de deformación \\
\hline Desplazamiento resultante & $\begin{array}{l}0 \mathrm{~mm} \\
\text { Nodo: } 1\end{array}$ & $\begin{array}{l}1,31654 \mathrm{~mm} \\
\text { Nodo: } 7.533\end{array}$ & 39 \\
\hline
\end{tabular}

10.2. RESULTADOS DEL ESTUDIO

10.2.1. DESPLAZAMIENTOS

Deformación máxima 1,32 mm.

10.2.2. FACTOR DE SEGURIDAD

$\begin{array}{cccc}\text { Tipo } & \text { Mín. } & \text { Máx. } & \text { Distribución de factor de seguridad } \\ \text { Automático } & 2,98368 & 431,557 & \text { FDS mín }=3 \\ & \text { Nodo: } 1 & \text { Nodo: } 169 & \end{array}$

Factor de seguridad 2,98.

\subsection{CONCLUSIÓN}

En base a los datos mostrados, podemos afirmar que la pantalla, en el caso más desfavorable, es capaz de resistir una fuerza de hasta $2.625,64 \mathrm{~N} / \mathrm{m}^{2}$ producida por viento siendo la fuerza estudiada, en base a la normativa aplicada, de $880 \mathrm{~N} / \mathrm{m}^{2}$, por lo tanto la resistencia es muy superior a la necesaria.

La deformación máxima a la fuerza aplicada es de 1,32 mm. 


\section{SIMULACIÓN DE LA PIEZA 3P.}

\subsection{PROPIEDADES DEL MATERIAL}

$$
\begin{array}{cc}
\text { Límite elástico: } & 300.000 \mathrm{~N} / \mathrm{m}^{2} \\
\text { Límite de compresión: } & 300.000 \mathrm{~N} / \mathrm{m}^{2} \\
\text { Módulo elástico: } & 1 \mathrm{e}+008 \mathrm{~N} / \mathrm{m}^{2} \\
\text { Coeficiente de Poisson: } & 0,35 \\
\text { Densidad: } & 1.680 \mathrm{~kg} / \mathrm{m}^{3}
\end{array}
$$

11.2. RESULTADOS DEL ESTUDIO

\subsubsection{DESPLAZAMIENTOS}

\section{Tipo Mín. Máx. Escala de deformación}

$\begin{array}{ccc}0 \text { Desplazamiento resultante } & \begin{array}{c}0 \mathrm{~mm} \\ \text { Nodo: } 1\end{array} & \begin{array}{c}0,921581 \mathrm{~mm} \\ \text { Nodo: } 7.533\end{array} 559,01\end{array}$

Deformación máxima menor de $1 \mathrm{~mm}$.

\subsubsection{FACTOR DE SEGURIDAD}

$\begin{array}{cccc}\text { Tipo } & \text { Mín. } & \text { Máx. } & \text { Distribución de factor de seguridad } \\ \text { Automático } & 3,89176 & 562,9 & \text { FDS mín }=3,9 \\ & \text { Nodo: } 1 & \text { Nodo: } 169 & \end{array}$

Factor de seguridad 3,89.

\subsection{CONCLUSIÓN}

En base a los datos mostrados, podemos afirmar que la pantalla, en el caso más desfavorable, es capaz de resistir una fuerza de hasta $3.424,75 \mathrm{~N} / \mathrm{m}^{2}$, producida por viento siendo la fuerza estudiada, en base a la normativa aplicada, de $880 \mathrm{~N} / \mathrm{m}^{2}$, por lo tanto la resistencia es muy superior a la necesaria.

La deformación máxima a la fuerza aplicada es menor que $1 \mathrm{~mm}$. 


\section{SIMULACIÓN DE LA PIEZA 4P.}

12.1. PROPIEDADES DEL MATERIAL

$\begin{array}{cc}\text { Límite elástico: } & 150.000 \mathrm{~N} / \mathrm{m}^{2} \\ \text { Límite de compresión: } & 150.000 \mathrm{~N} / \mathrm{m}^{2} \\ \text { Módulo elástico: } & 4 \mathrm{e}+007 \mathrm{~N} / \mathrm{m}^{2} \\ \text { Coeficiente de Poisson: } & 0,35 \\ \text { Densidad: } & 1.680 \mathrm{~kg} / \mathrm{m}^{3}\end{array}$

12.2. RESULTADOS DEL ESTUDIO

12.2.1. DESPLAZAMIENTOS

Tipo Mín. Máx. Escala de deformación

$\begin{array}{llll}\text { Desplazamiento resultante } & \begin{array}{c}0 \mathrm{~mm} \\ \text { Nodo: } 1\end{array} & \begin{array}{l}4,26671 \mathrm{~mm} \\ \text { Nodo: } 7.533\end{array} 112,555\end{array}$

Deformación máxima 4,27 mm.

\subsubsection{FACTOR DE SEGURIDAD}

$\begin{array}{cccc}\text { Tipo } & \text { Mín. } & \text { Máx. } & \text { Distribución de factor de seguridad } \\ \text { Automático } & 1,50332 & 1.267,73 & \text { FDS mín }=1,5 \\ & \text { Nodo: } 1 & \text { Nodo: } 401 & \end{array}$

Factor de seguridad 1,5.

\subsection{CONCLUSIÓN}

En base a los datos mostrados, podemos afirmar que la pantalla, en el caso más desfavorable, es capaz de resistir una fuerza de hasta $1.322,92 \mathrm{~N} / \mathrm{m}^{2}$, producida por viento siendo la fuerza estudiada, en base a la normativa aplicada, de $880 \mathrm{~N} / \mathrm{m}^{2}$, por lo tanto la resistencia es muy superior a la necesaria.

La deformación máxima a la fuerza aplicada es de 4,27 mm. 


\section{SIMULACIÓN DE LA PIEZA 5P.}

13.1. PROPIEDADES DEL MATERIAL

\begin{tabular}{cc} 
Límite elástico: & $230.000 \mathrm{~N} / \mathrm{m}^{2}$ \\
\hline Límite de compresión: & $225.000 \mathrm{~N} / \mathrm{m}^{2}$ \\
Módulo elástico: & $7 \mathrm{e}+007 \mathrm{~N} / \mathrm{m}^{2}$ \\
Coeficiente de Poisson: & 0,35 \\
Densidad: & $1.680 \mathrm{~kg} / \mathrm{m}^{3}$
\end{tabular}

13.2. RESULTADOS DEL ESTUDIO

13.2.1. DESPLAZAMIENTOS

Tipo

Desplazamiento resultante
Mín.

$0 \mathrm{~mm}$

Nodo: 1
Máx. 2,43812 $\mathrm{mm}$

Nodo: 7.533
Escala de deformación

196,971

Deformación máxima 2,44mm.

\subsubsection{FACTOR DE SEGURIDAD}

Tipo Mín. Máx. Distribución de factor de seguridad

Automático 2,30508 1.943,86

Nodo: 1 Nodo: 401

FDS mín $=2,3$

Factor de seguridad 2,30.

\subsection{CONCLUSIÓN}

En base a los datos mostrados, podemos afirmar que la pantalla, en el caso más desfavorable, es capaz de resistir una fuerza de hasta $2.028,47 \mathrm{~N} / \mathrm{m}^{2}$, producida por viento siendo la fuerza estudiada, en base a la normativa aplicada, de $880 \mathrm{~N} / \mathrm{m}^{2}$, por lo tanto la resistencia es muy superior a la necesaria.

La deformación máxima a la fuerza aplicada es de 2,44 mm. 


\section{SIMULACIÓN DE LA PIEZA 6P.}

14.1. PROPIEDADES DEL MATERIAL

$\begin{array}{cc}\text { Límite elástico: } & 300.000 \mathrm{~N} / \mathrm{m}^{2} \\ \text { Límite de compresión: } & 300.000 \mathrm{~N} / \mathrm{m}^{2} \\ \text { Módulo elástico: } & 1 \mathrm{e}+008 \mathrm{~N} / \mathrm{m}^{2} \\ \text { Coeficiente de Poisson: } & 0,35 \\ \text { Densidad: } & 1.680 \mathrm{~kg} / \mathrm{m}^{3}\end{array}$

14.2. RESULTADOS DEL ESTUDIO

14.2.1. DESPLAZAMIENTOS

Tipo

Desplazamiento resultante
Mín. Máx.

$0 \mathrm{~mm} \quad 1,70668 \mathrm{~mm}$

Nodo: 1 Nodo: 7.533
Escala de deformación

281,388

Deformación máxima 1,7 mm.

\subsubsection{FACTOR DE SEGURIDAD}

$\begin{array}{cccc}\text { Tipo } & \text { Mín. } & \text { Máx. } & \text { Distribución de factor de seguridad } \\ \text { Automático } & 3,00663 & 2.535,46 & \text { FDS mín }=3 \\ & \text { Nodo: } 1 & \text { Nodo: } 401 & \end{array}$

Factor de seguridad 3.

\subsection{CONCLUSIÓN}

En base a los datos mostrados, podemos afirmar que la pantalla, en el caso más desfavorable, es capaz de resistir una fuerza de hasta 2.645,83 $\mathrm{N} / \mathrm{m}^{2}$, producida por viento siendo la fuerza estudiada, en base a la normativa aplicada, de $880 \mathrm{~N} / \mathrm{m}^{2}$, por lo tanto la resistencia es muy superior a la necesaria.

La deformación máxima a la fuerza aplicada es de 1,7 mm. 


\section{SIMULACIÓN DE LA PIEZA 7P.}

Con cargas por viento. Densidad $1.200 \mathrm{~kg} / \mathrm{m}^{3}$, se considera que ha superado el punto de saturación de agua.

15.1. PROPIEDADES DEL MATERIAL

$$
\begin{array}{cc}
\text { Límite elástico: } & 800.000 \mathrm{~N} / \mathrm{m}^{2} \\
\text { Módulo elástico: } & 8 \mathrm{e}+006 \mathrm{~N} / \mathrm{m}^{2} \\
\text { Coeficiente de Poisson: } & 0,35 \\
\text { Densidad: } & 1.200 \mathrm{~kg} / \mathrm{m}^{3}
\end{array}
$$

15.2. RESULTADOS DEL ESTUDIO

\subsubsection{DESPLAZAMIENTOS}

\begin{tabular}{cccc} 
Tipo & Mín. & Máx. & Escala de deformación \\
Desplazamiento resultante & $0 \mathrm{~mm}$ & $7,03295 \mathrm{~mm}$ & 67,2061 \\
\cline { 2 - 3 } & Nodo: 1 & Nodo: 7.533 &
\end{tabular}

Deformación máxima $7 \mathrm{~mm}$.

\subsubsection{FACTOR DE SEGURIDAD}

Tipo Mín. Máx. Distribución de factor de seguridad

Automático $15,43 \quad 2.067 \quad$ FDS mín $=15$

Factor de seguridad 15.

\subsection{CONCLUSIÓN}

En este ensayo la fuerza del viento es $880 \mathrm{~N} / \mathrm{m}^{2}$.

La deformación máxima a la fuerza aplicada es de $7 \mathrm{~mm}$ en la parte superior trasera.

No hay afección importante por lluvia excesiva y viento excesivo. 


\section{SIMULACIÓN DE LA PIEZA 8P.}

Con cargas por viento. Densidad $1.200 \mathrm{~kg} / \mathrm{m}^{3}$, se considera que ha superado el punto de saturación de agua.

16.1. PROPIEDADES DEL MATERIAL

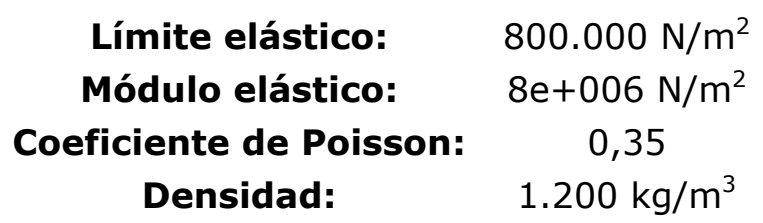

16.2. RESULTADOS DEL ESTUDIO

16.2.1. DESPLAZAMIENTOS

\begin{tabular}{cccc} 
Tipo & Mín. & Máx. & Escala de deformación \\
Desplazamiento resultante & $0 \mathrm{~mm}$ & $16,7535 \mathrm{~mm}$ & 28,1188 \\
\cline { 2 - 3 } & Nodo: 1 & Nodo: 7.533 &
\end{tabular}

Deformación máxima 16,75mm.

16.2.2. FACTOR DE SEGURIDAD

$\begin{array}{cccc}\text { Tipo } & \text { Mín. } & \text { Máx. } & \text { Distribución de factor de seguridad } \\ \text { Automático } & 10,7333 & 8.714,88 & \text { FDS mín }=11 \\ & \text { Nodo: } 1 & \text { Nodo: } 9.994 & \end{array}$

Factor de seguridad 11.

\subsection{CONCLUSIÓN}

En este ensayo hay fuerza del viento $880 \mathrm{~N} / \mathrm{m}^{2}$.

La deformación máxima a la fuerza aplicada es de $16,75 \mathrm{~mm}$ en la parte superior trasera.

No hay afección importante por lluvia excesiva y viento excesivo. 


\title{
17. SIMULACIÓN DE LA PIEZA 9P.
}

Con cargas por viento. Densidad $1.200 \mathrm{~kg} / \mathrm{m}^{3}$, se considera que ha superado el punto de reblandecimiento por sobresaturación de agua.

\subsection{PROPIEDADES DEL MATERIAL}

\author{
Límite elástico: $\quad 150.000 \mathrm{~N} / \mathrm{m}^{2}$ \\ Módulo elástico: $\quad 3 e+006 \mathrm{~N} / \mathrm{m}^{2}$ \\ Coeficiente de Poisson: $\quad 0,35$ \\ Densidad: $\quad 1.200 \mathrm{~kg} / \mathrm{m}^{3}$
}

\subsection{RESULTADOS DEL ESTUDIO}

\subsubsection{DESPLAZAMIENTOS}

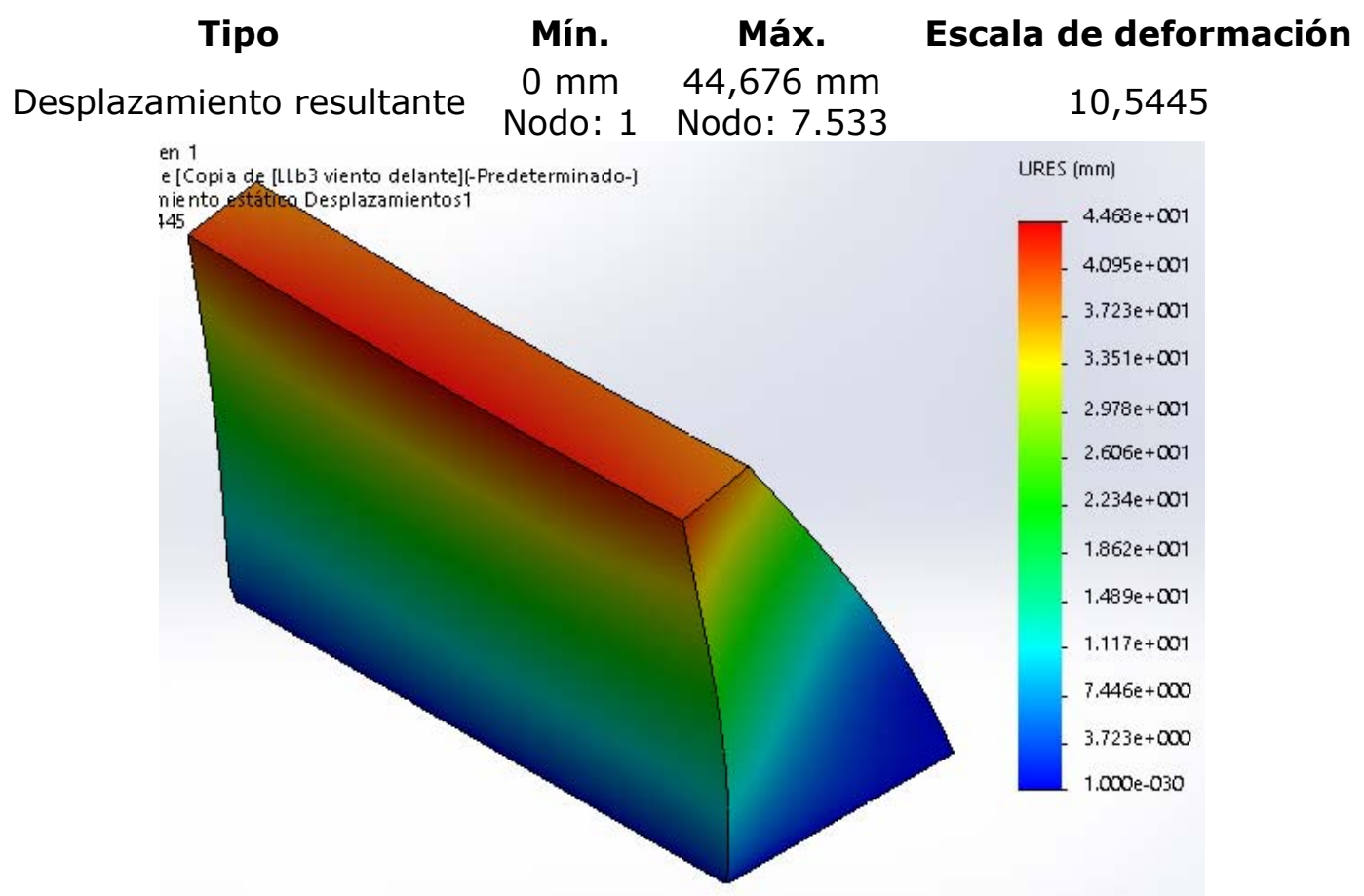

Deformación máxima 44,68mm. 


\subsubsection{FACTOR DE SEGURIDAD}

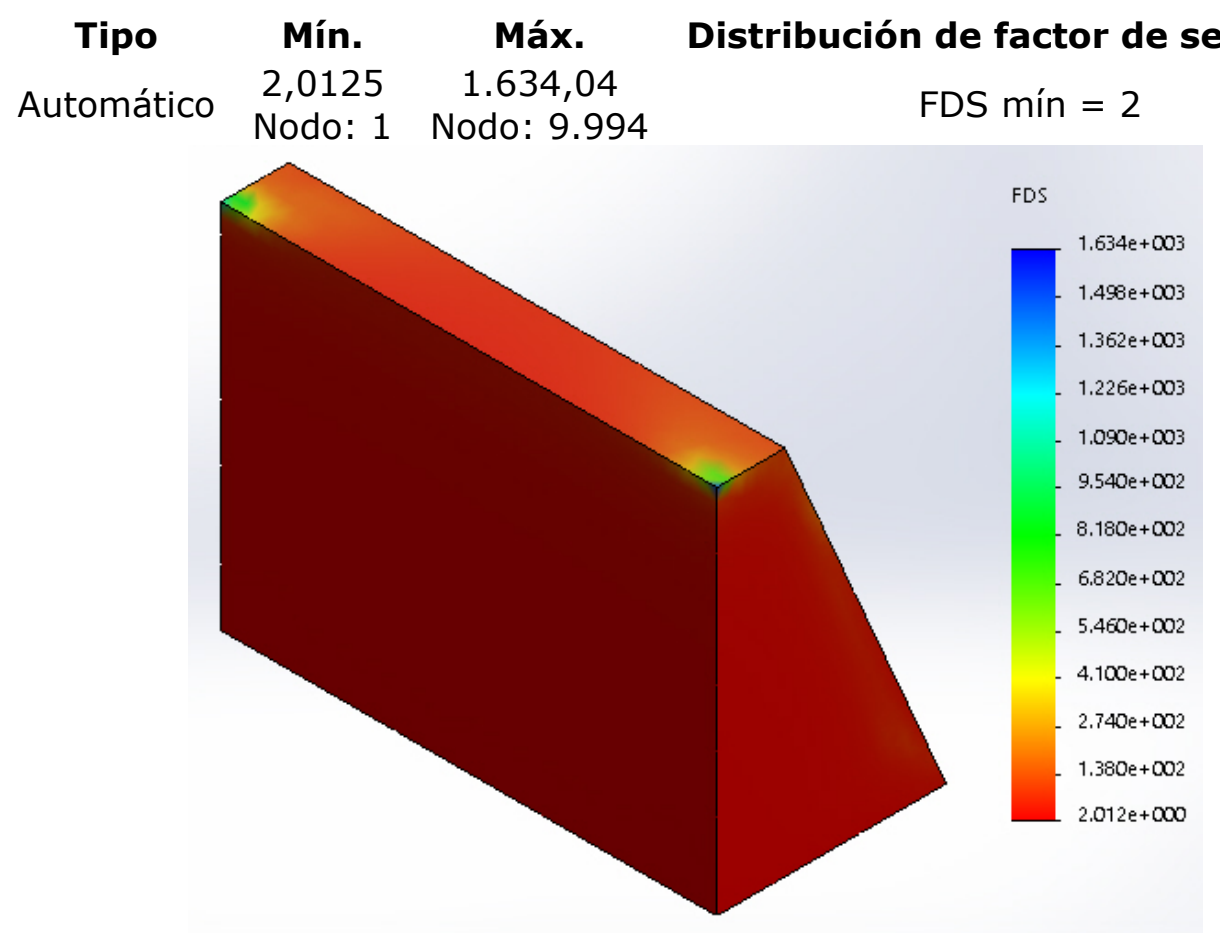

Factor de seguridad 2.

\subsection{CONCLUSIÓN}

En base a los datos mostrados, podemos afirmar que la pantalla, en el caso más desfavorable, es capaz de resistir una fuerza de hasta $1.771 \mathrm{~N} / \mathrm{m}^{2}$, producida por viento siendo la fuerza estudiada, en base a la normativa aplicada, de $880 \mathrm{~N} / \mathrm{m}^{2}$, por lo tanto la resistencia es muy superior a la necesaria.

La deformación máxima a la fuerza aplicada es de 44,68 mm.

\section{CONCLUSIONES FINALES}

La pantalla en seco tiene una buena estabilidad frente a su propia forma y frente a las fuerzas exteriores como el viento. La arcilla mezclada con el molido de jardinería y con poca humedad casi no sufre deformación al aplicarle $220 \mathrm{~kg}$ de peso sobre una superficie de $0,11 \mathrm{~m}^{2}$.

Medidas límite: Factor de seguridad de 1,5 en la pieza 4P y deformación máxima de $16,75 \mathrm{~mm}$ en la pieza $8 \mathrm{P}$.

Al aumentar el grado de humedad tiene un buen comportamiento hasta llegar a la saturación que pasa a comportarse como plástico. En Gran Canaria, en la zona de costa, las lluvias suelen ser ocasionales y de poca duración. Para que la pantalla se sature se requiere que la lluvia dure más de un día, ya que la arcilla mezclada tiene una cierta resistencia a la penetración del agua. 
Si por seguridad se requiere reducir la posible deformación plástica de la pantalla por saturación se puede corregir enjaulándola con mallazo, retícula metálica en la que las varillas de acero están soldadas en los puntos de intersección.

\section{BIBLIOGRAFÍA}

BAÑóN Blázquez, Luis y BEVIÁ García, José Francisco (2000). Manual de carreteras. Volumen II: Construcción y mantenimiento. Editorial Enrique Ortiz e Hijos, Contratista de obras S.A. Alicante.

IBÁÑNEZ, Juan José. EL agua en el Suelo 4: Textura del Suelo y Propiedades Hídricas. MadrI+D Fundación para el Conociemiento. Consejería de Educación, Juventud y Deporte, Comunidad de Madrid, 2006 [En línea] Consultado en

web: http://www.madrimasd.org/blogs/universo/2006/07/05/33887.

Fecha de consulta: Noviembre, 2015.

Instituto Eduardo Torroja de Ciencias de la Construcción, con la colaboración de CEPCO y AICIA (2010). Catálogo de Elementos Constructivos. Ministerio de Vivienda.

ISO 9613-2 Acoustics - Attenuation of sound during propagation outdoors, 1996.

Ministerio de Fomento (2009). Documento Básico, S. E. A. E. Seguridad Estructural. Acciones en la Edificación. Código Técnico de la Edificación, pág. 7-8.

MUELAS Rodríguez, Ángel. Manual de mecánica del suelo y cimentaciones. [En línea] Consultada en web:

http://www.uned.es/dpto-icf/mecanica_del_suelo_y_cimentaciones/images/ mecansueloycimentaciones_anejoA.pdf. Fecha de consulta: Septiembre, 2015.

SOSA, P. A.; NARANJO, A.; MÁRQUEZ, M.; ESCANDELL, A. y GONZÁLEZ Pérez, M. Á (2007). Atlas de los palmerales de Gran Canaria. Obra Social de la Caja de Canarias. 